\title{
Preemptive pyloroplasty for iatrogenic vagus nerve injury in intrahepatic cholangiocarcinoma patients undergoing extensive left-sided lymph node dissection: a retrospective observational study
}

\author{
Shin Hwang, Dong-Hwan Jung, Eun-Kyoung Jwa, Yumi Kim \\ Division of Hepatobiliary Surgery, Department of Surgery, Asan Medical Center, University of Ulsan College of Medicine, Seoul, Korea
}

\begin{abstract}
Background: Intrahepatic cholangiocarcinoma (ICC) of the left liver often shows left-sided lymph node (LN) metastasis. If gastric lesser curvature is extensively dissected, it can induce an iatrogenic injury to the extragastric vagus nerve branches that control motility of the pyloric sphincter and lead to gastric stasis. To cope with such LN dissection-associated gastric stasis, we performed pyloroplasty preemptively. The objective of this study was to analyze our 20-year experience of preemptive pyloroplasty performed in 10 patients. Methods: We investigated clinical sequences of 10 patients with ICC who underwent preemptive pyloroplasty following left hepatectomy and extended left-sided LN dissection. Incidence of gastric stasis and oncological survival outcomes were analyzed.

Results: All 10 patients were classified as stage IIIB due to T1-3N1M0 stage according to the 8th edition of American Joint Committee on Cancer staging system. The overall patient survival rate was $51.9 \%$ at 1 year, $25.9 \%$ at 2 years, and $0 \%$ at 3 years. Seven patients showed uneventful postoperative recovery after surgery. Two patients suffered from gastric stasis, which was successfully managed with supportive care. One patient suffered from overt gastric paresis, which was successfully managed with azithromycin administration for 1 month.

Conclusion: We believe that preemptive pyloroplasty is an effective surgical option to prevent gastric stasis in patients undergoing extensive left-sided LN dissection. Azithromycin appears to be a potent prokinetic agent in gastroparesis.
\end{abstract}

Keywords: Gastric emptying; Gastroparesis; Pylorus; Vagus nerve

\section{Introduction}

Frequent sites of lymph node (LN) metastasis in patients with left-sided intrahepatic cholangiocarcinoma (ICC) include the common hepatic artery, celiac axis, and even the lesser curvature of the stomach, thus named as left-sided LN metastasis [1,2]. If metastatic LNs are located at the lesser curvature of the stomach, the connective tissues over the gastric serosa at the lesser curvature side should be dissected to ensure complete LN dissection. Such surgical procedure inevitably results in iatrogenic vagus nerve injury because the running course of this nerve toward the pylorus and antrum is interrupted by extensive LN dissection, by which vagotomy-associated gastric stasis can occur. We have previously reported that preemptive pyloroplasty is effective in preventing such LN dissection-associated gastric stasis [3]. The objective of the present study was to analyze the effect of preemptive pyloro-

Received: October 12, 2021 • Revised: November 17, 2021 • Accepted: November 17, 2021

Corresponding author: Shin Hwang, MD, PhD, FACS

Department of Surgery, Asan Medical Center, University of Ulsan College of Medicine, 88 Olympic-ro 43-gil, Songpa-gu, Seoul 05505, Korea

Tel: +82-2-3010-3930•Fax: +82-2-3010-6701・E-mail: shwang@amc.seoul.kr 
plasty in 10 patients who underwent preemptive pyloroplasty during left hepatectomy combined with extensive $\mathrm{LN}$ dissection for left-sided ICC.

\section{Methods}

Ethical statements: The study protocol was approved by the Institutional Review Board (IRB) of Asan Medical Center (IRB No: 2021-1347). The requirement for informed consent from patients was waived due to the retrospective nature of this study. This study was performed in accordance with the ethical guidelines of the World Medical Association Declaration of Helsinki 2013.

\section{Patient selection}

The liver cancer database of our institution was searched to identi- fy patients who underwent left hepatectomy or left lateral sectionectomy combined with extensive $\mathrm{LN}$ dissection for left-sided ICC. During the 20-year study period from January 2001 to June 2021, 10 patients underwent preemptive Heineke-Mikulicz pyloroplasty to prevent iatrogenic vagus nerve injury-associated gastric stasis. These 10 patients were selected as the study group in the present study.

The study patients were followed until August 2021 through institutional medical record review and assistance of National Health Insurance Service.

\section{Decision on whether to perform preemptive Heineke- Mikulicz pyloroplasty}

After completion of left-sided hepatectomy and extensive LN dissection for left-sided LN metastasis (Fig. 1), we decided whether to perform preemptive pyloroplasty after careful inspection of the gastric serosa at the lesser curvature. If the gastric serosa was fully
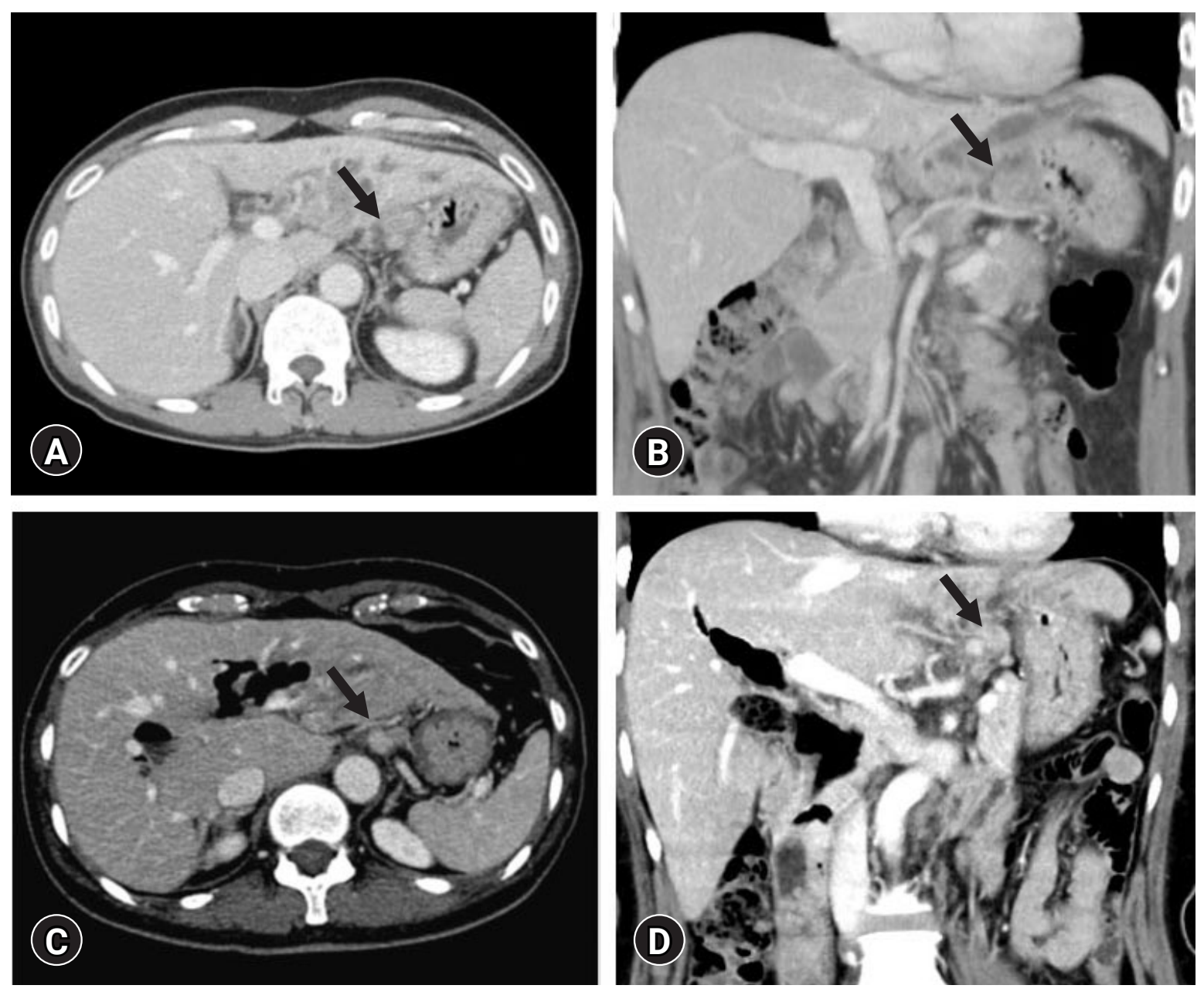

Fig. 1. Preoperative abdominal computed tomography of patients with left-sided intrahepatic cholangiocarcinoma. $(A, B)$ Regional lymph node metastasis is identified around the lesser curvature of the stomach (arrows) in a 59-year-old male patient and (C, D) a 57-year-old female patient. 

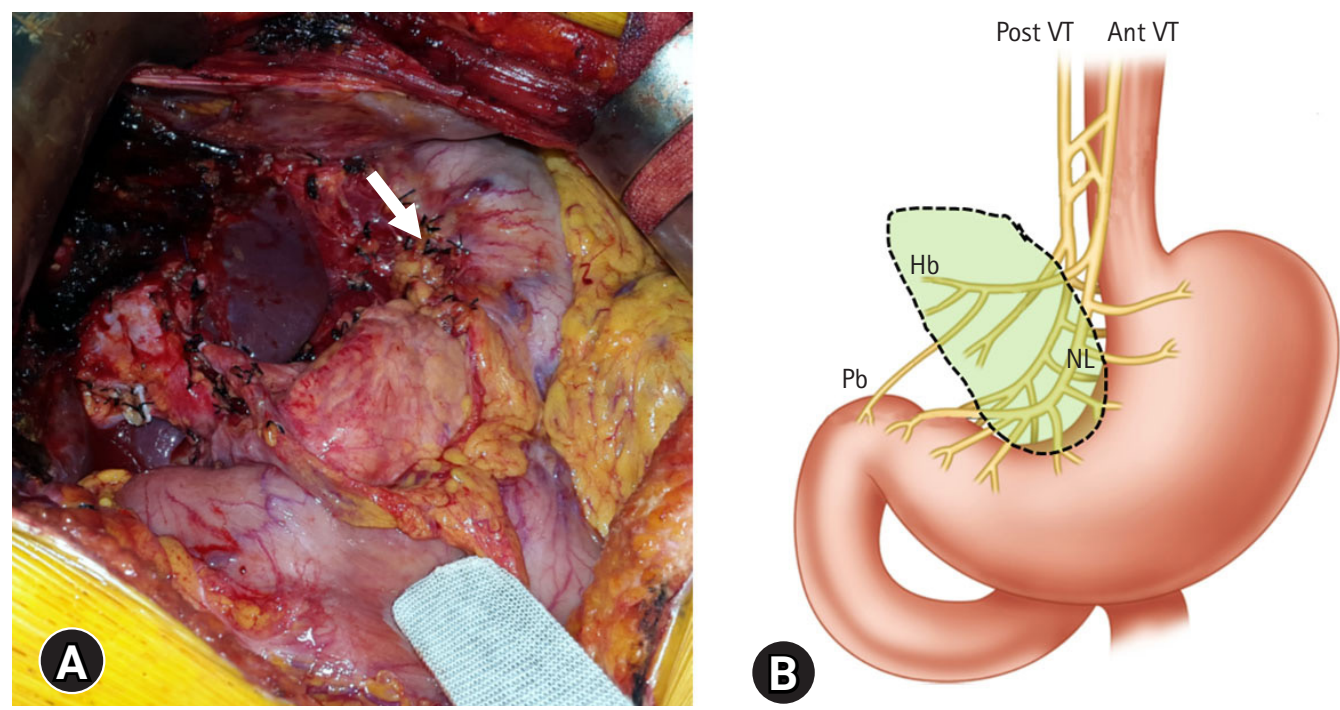

Fig. 2. The extent of vagus nerve injury according to extensive left-sided lymph node dissection. (A) Intraoperative photograph showing denuding of the serosa (arrow) at the lesser curvature of the stomach. (B) Illustration of the extragastric vagus nerve innervation. The encircled area indicates the extent of vagus nerve injury. Post $\mathrm{VT}$, the posterior vagus nerve trunk; Ant $\mathrm{VT}$, anterior vagus nerve trunk; NL, the nerve of Latarjet; $\mathrm{Hb}$, hepatic branches; $\mathrm{Pb}$, pyloric branches.

exposed with clearing of the overlying connective tissues and feeding vessels, the vagus nerve branch toward the pylorus and antrum was determined to be transected. The denervated extent by the vagus nerve transection was comparable to that of selective vagotomy (Fig. 2). Thus, such condition was reasonably indicated for preemptive pyloroplasty [3]. We performed the classical hand-sewing pyloroplasty of Heinke-Mikulicz type. The wall of the distal antrum and the duodenal first portion was longitudinally incised with electrocautery and then transversely repaired with inner continuous sutures using 4-0 absorbable monofilament and outer continuous sutures using 5-0 nonabsorbable monofilament or interrupted sutures with 3-0 or 4-0 black silk (Fig. 3).

\section{Postoperative assessment for gastric stasis}

A nasogastric tube (NGT) was routinely kept under natural drainage for 3 days after surgery in all patients who had undergone preemptive pyloroplasty. If gastric stasis of any degree was suspected, the NGT was kept and frequently aspirated until recovery of gastrointestinal motility. The NGT was removed if the amount of daily drainage was less than $300 \mathrm{~mL} /$ day with absence of air-fluid level at the stomach on simple abdominal X-ray. During fasting, all patients received total parenteral nutrition until intake of soft diet. Prokinetics were administered with temporary clamping of the NGT with an expectation to facilitate recovery of bowel motility $[4,5]$.

\section{Postoperative treatment and surveillance}

All patients underwent adjuvant chemotherapy with or without concurrent chemoradiation therapy. General principles of treat- ment for stage IIIB ICC according to the 8th edition of American Joint Committee on Cancer (AJCC) staging system were applied. Detailed follow-up protocol was presented previously [6].

\section{Statistical analysis}

Numerical data are reported as mean with standard deviation. Survival curve was estimated with the Kaplan-Meier method. All statistical analyses were performed using IBM SPSS ver. 22 (IBM Corp., Armonk, NY, USA).

\section{Results}

\section{Patient demographics and survival outcomes}

All 10 patients were classified as stage IIIB according to the 8th edition of AJCC staging system because of T1-3N1M0 stage. Their clinicopathological profiles are summarized in Table 1 . The overall patient survival rate was $51.9 \%$ at 1 year, $25.9 \%$ at 2 years, and $0 \%$ at 3 years (Fig. 4).

\section{Incidence of gastric stasis}

For eight patients, the NGT was kept for the first 3 days after surgery. For the remaining two patients, it was kept for 4 days after surgery. One day after NGT removal, sips of water were initiated. Thereafter, intake of liquid diet was started after careful physical examination and daily simple abdominal $\mathrm{X}$-ray follow-up.

Seven patients showed no evidence of gastric stasis. Thus, diet intake was started at 5 days after surgery. No gastrointestinal motility disorder occurred until hospital discharge. 

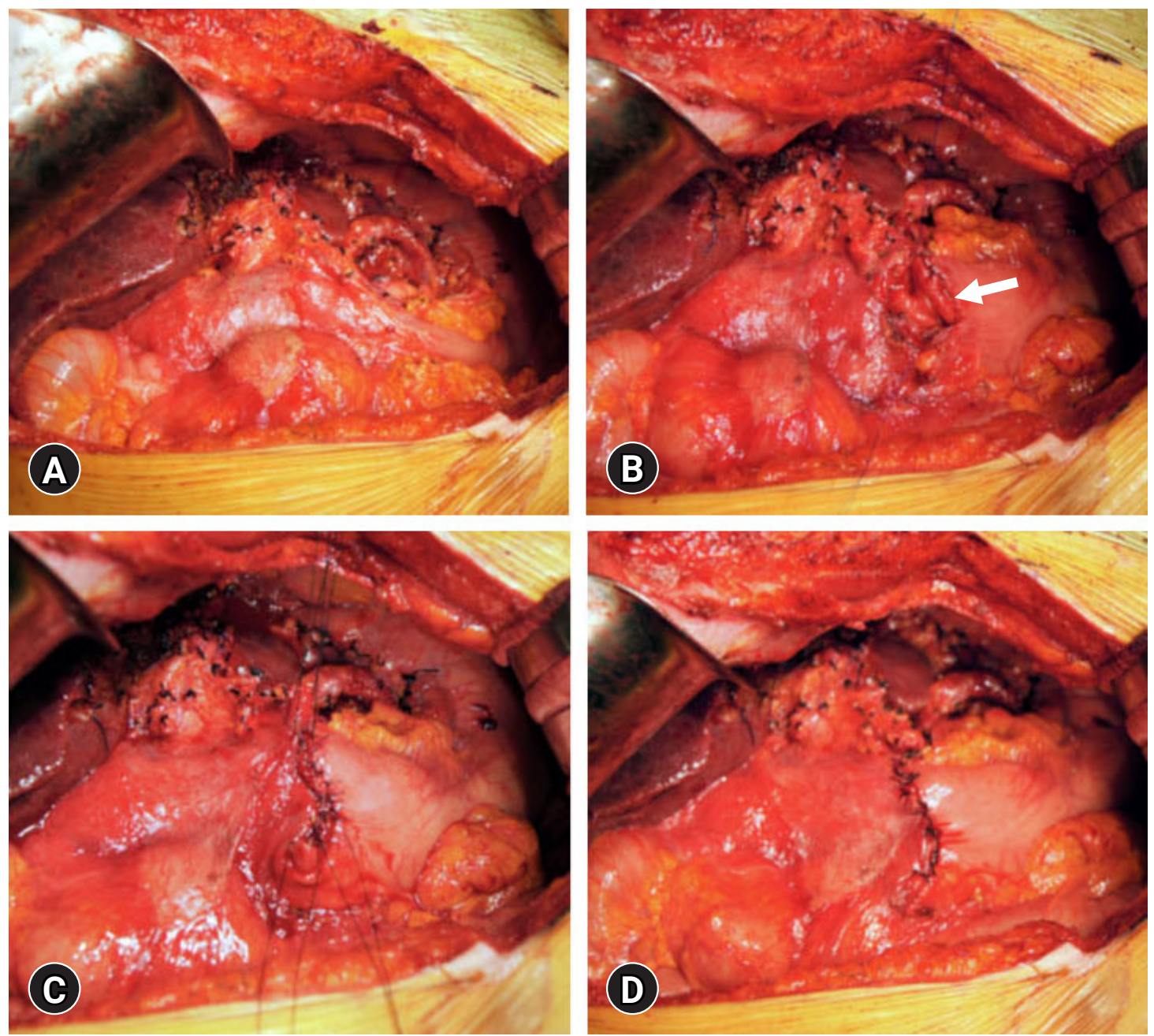

Fig. 3. Intraoperative procedure for Heineke-Mikulicz pyloroplasty. (A) The lesser curvature of the stomach is extensively dissected. (B) The wall of the distal antrum and the duodenal first portion is longitudinally incised (arrow) with electrocautery. (C) The incised wound is transversely repaired by inner continuous sutures using 4-0 absorbable monofilament. (D) The serosa is closed with 3-0 black silk sutures.

In contrast, the remaining three patients manifested clinical findings suggesting gastric stasis or paresis. Three patients showed gaseous distension of the stomach and air-fluid level in simple abdominal X-ray in the next few days after removal of the NGT. Thus, the early postoperative incidence of gastric motility disorder after preemptive pyloroplasty was $30 \%$.

\section{Clinical sequences of two patients showing gastric stasis}

A 61-year-old male patient who showed gastric stasis and paralytic ileus was well tolerated with fasting for 7 days after removal of the NGT without reinsertion of an NGT. Gastric distension and paralytic ileus slowly resolved. Diet intake was initiated 11 days after surgery and continued uneventfully until hospital discharge.

A 67-year-old male patient showed prolonged gastric distension and paralytic ileus after removal of the NGT, thus a new NGT tube was reinserted at 8 days after surgery and two prokinetic agents (gasmotin and ganakhan) were orally administered for 7 days until daily drainage of less than $200 \mathrm{~mL}$ / day with resolution of paralytic ileus pattern on simple abdominal X-ray follow-up. Diet intake was initiated 15 days after surgery and continued uneventfully until hospital discharge.

\section{Clinical sequences of one patient showing gastric pare-} sis

A 57-year-old female patient underwent left hepatectomy, choledochal cyst excision, and Roux-en-Y hepaticojejunostomy due to choledochal cyst-associated ICC (Fig. 1B, Fig. 5). The patient showed marked gaseous distension of the stomach with air-fluid level on simple abdominal X-ray (Fig. 6A, 6B) since removal of the NGT at 4 days after surgery. Two prokinetic agents (gasmotin and ganakhan) were orally administered, but they were ineffective. An NGT tube was reinserted at 9 days after surgery, but the amount 
Table 1. Clinicopathological features of patients with intrahepatic cholangiocarcinoma and left-sided lymph node metastasis

\begin{tabular}{|c|c|}
\hline Variable & Data \\
\hline Age (yr) & $57.3 \pm 7.9$ \\
\hline \multicolumn{2}{|l|}{$\operatorname{Sex}(n)$} \\
\hline Male & 4 \\
\hline Female & 6 \\
\hline ICG- $\mathrm{R}_{15}(\%)$ & $10.3 \pm 4.3$ \\
\hline \multicolumn{2}{|l|}{ Extent of hepatic resection (n) } \\
\hline Left hepatectomy & 9 \\
\hline Left lateral sectionectomy & 1 \\
\hline Concurrent bile duct resection (n) & 1 \\
\hline Tumor size $(\mathrm{cm})$ & $7.1 \pm 4.2$ \\
\hline \multicolumn{2}{|l|}{ Perineural invasion (n) } \\
\hline Absent & 2 \\
\hline Present & 8 \\
\hline \multicolumn{2}{|l|}{ Lymphovascular invasion (n) } \\
\hline Absent & 2 \\
\hline Present & 8 \\
\hline \multicolumn{2}{|l|}{ Tumor differentiation (n) } \\
\hline Well differentiated & 0 \\
\hline Moderately differentiated & 7 \\
\hline Poorly differentiated & 3 \\
\hline \multicolumn{2}{|l|}{ Tumor growth pattern (n) } \\
\hline Mass-forming & 7 \\
\hline Periductal infiltrating & 3 \\
\hline \multicolumn{2}{|l|}{ 7th AJCC tumor stage (n) } \\
\hline $\mathrm{T} 1$ & 3 \\
\hline $\mathrm{T} 2$ & 6 \\
\hline T3 & 1 \\
\hline $\mathrm{T} 4$ & 0 \\
\hline Incidence of gastric motility disorder & 3 \\
\hline
\end{tabular}

Values are presented as mean \pm standard deviation or number only. ICG- $\mathrm{R}_{15}$, indocyanine green retention test at 15 minutes; AJCC, American Joint Committee on Cancer.

of daily drainage was less than $200 \mathrm{~mL} /$ day despite overt gaseous distension of the stomach and persistence of air-fluid level at the stomach. Thus, the NGT was repeatedly aspirated every 4 hours, which resulted in daily drainage of more than $1,000 \mathrm{~mL} /$ day. For 10 days of NGT drainage, gastric distension did not resolve at all. Oral administration of bethanechol was ineffective. On the postoperative 20th day, upper gastrointestinal series with gastrograffin showed delayed gastric emptying (Fig. 6C, 6D), indicating gastric paresis rather than gastric outlet obstruction. We presumed that the cause of such overt gastric paresis was denervation of the vagus nerve.

On the postoperative 21st day, azithromycin $500 \mathrm{mg}$ was administered intravenously once a day. On the next day, the patient felt the loss of epigastric discomfort and NGT tube drainage decreased

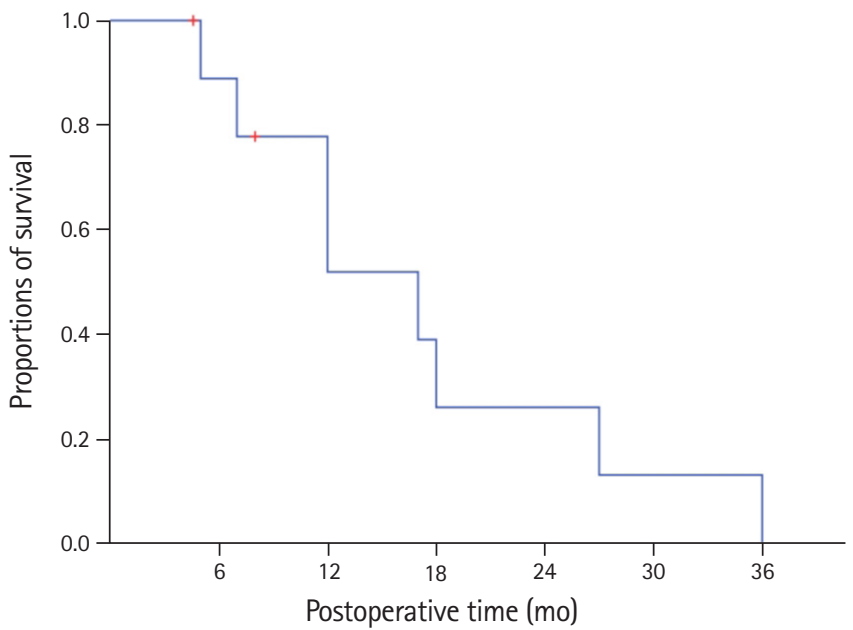

Fig. 4. Kaplan-Meier overall patient survival curve. Red cross markers indicate censored cases.

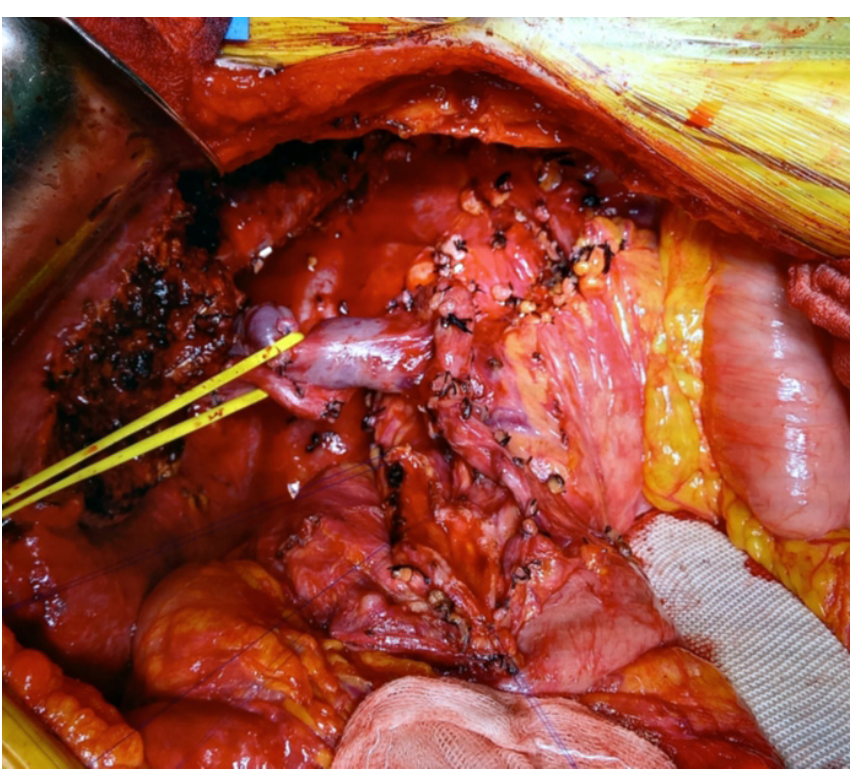

Fig. 5. Intraoperative photograph of a 57-year-old patient undergoing left hepatectomy and choledochal cyst excision.

markedly. After intravenous administration of azithromycin for 3 days, the NGT tube was removed. Endoscopic gastroduodenoscopy showed a slight narrowing of the duodenum probably due to extrinsic compression, thus balloon dilatation was performed. Diet intake was initiated with oral administration of azithromycin and two prokinetics, and oral intake was progressed uneventfully. The patient was discharged after azithromycin administration for 8 days on the postoperative 29th day with uneventful oral intake (Fig. 7A, 7B). One prokinetic agent was stopped 1 week after discharge due to diarrhea and another agent was also discontinued 1 week later. Diarrhea disappeared after discontinuation of prokinetics (Fig. 7C, 7D). Azithromycin was discontinued after overall 4 weeks of use. 

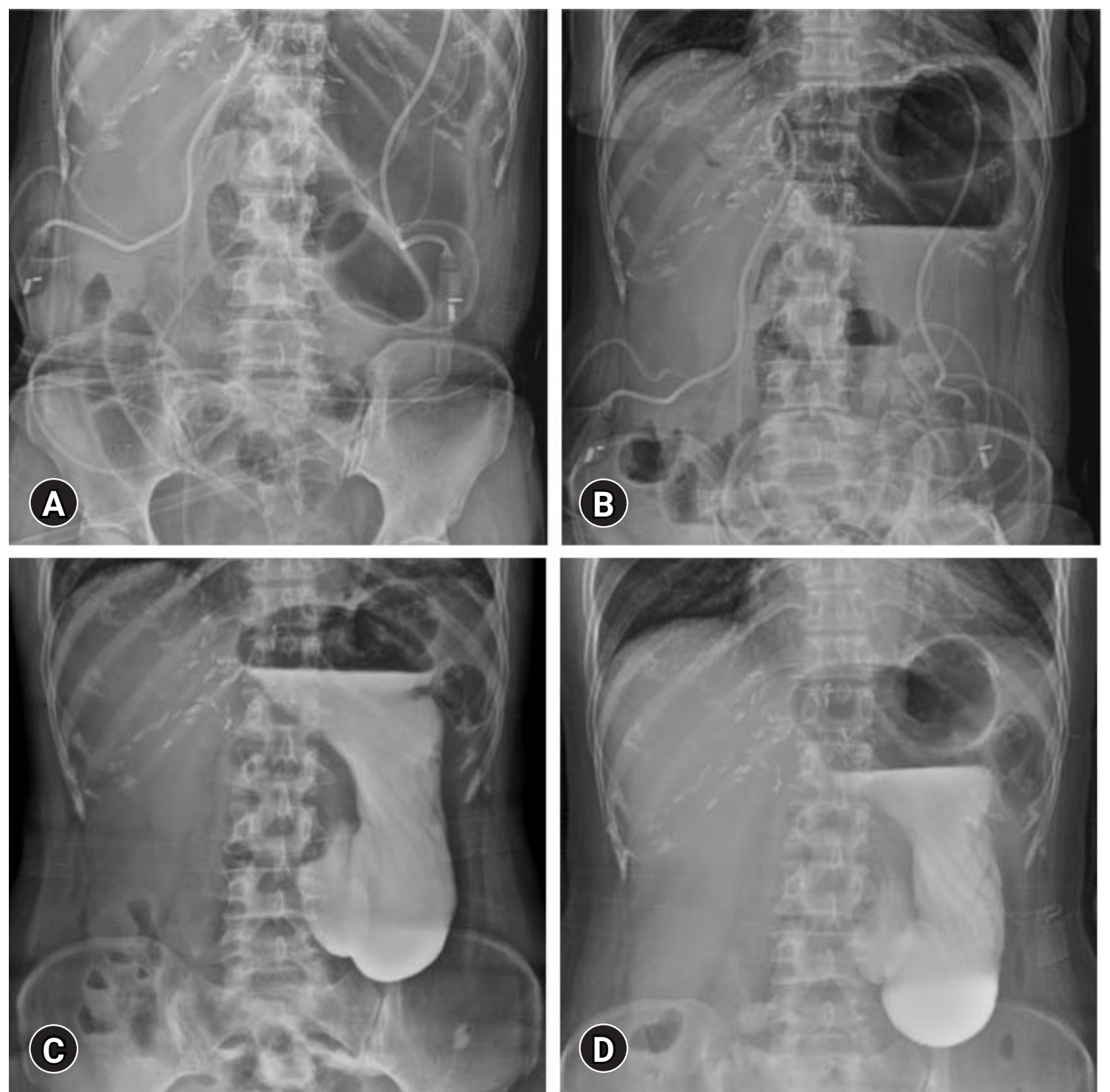

Fig. 6. Postoperative simple abdominal X-ray images follow-up. (A, B) Images taken on the postoperative 8th day show marked gaseous distension of the stomach with air-fluid level. (C, D) Images taken on the postoperative 20th day show stagnated gastrograffin within the stomach at (C) 1 hour and (D) 6 hours after oral intake.

Thereafter, bowel motility was well maintained without any medication. The patient has undergone the scheduled adjuvant chemotherapy for 6 months without delay.

\section{Discussion}

ICC is the second most common primary malignancy of the liver. Its diagnosis has been gradually rising in the general population. ICC comprises approximately $15 \%$ of primary liver malignancies in patients undergoing surgical resection in our institution [6].

LN metastasis is known to be the most potent prognostic factor in patients undergoing surgical resection for ICC [6-8]. We have previously presented that $\mathrm{LN}$ metastasis and perineural invasion are independent risk factors for ICC [6]. LN metastasis alone makes the tumor stage IV in the 7th AJCC staging system and tumor stage IIIB in the 8th AJCC staging system for ICC. Presence of LN metastasis is associated with very poor outcomes. The clinical benefit of aggressive LN dissection has not been unclearly defined yet, but it has been regarded as an essential part of resectional surgery for ICC [8-11].

If left-sided LN metastasis is identified, the usual extent of LN dissection may include the hepatoduodenal ligament, common hepatic artery, celiac axis, left gastric artery, and lesser curvature of the stomach. If an $\mathrm{LN}$ is located close to the lesser curvature of the stomach, its dissection procedure may include clearing of the gastric lesser curvature and ligation of the left gastric artery. This surgi- 

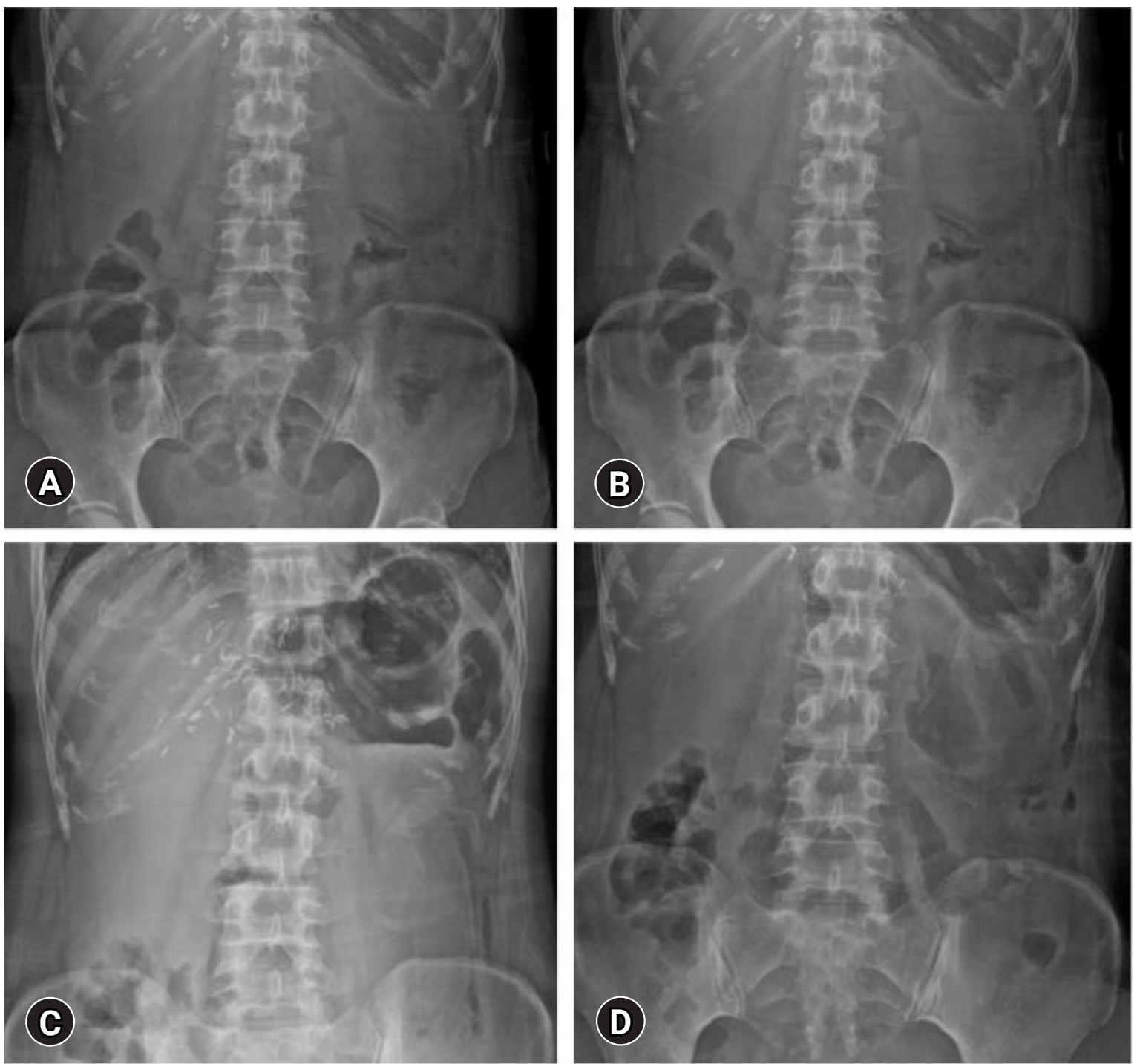

Fig. 7. Postoperative simple abdominal X-ray follow-up images after azithromycin administration. The images taken at (A, B) hospital discharge and (C, D) 2 weeks later show no significant abnormal finding.

cal procedure can induce an iatrogenic injury to the vagus nerve branches which control motility of the pyloric sphincter, by which intractable gastric stasis can occur. Denervation of the controlling vagus nerve branches to the antrum and pylorus can manifest delay of gastric emptying, which persists up 1 to 2 months, as presented in our previous study [3].

Not to mention LN dissection, left hepatectomy per se is known to be associated with disorders of gastric motility. After living-donor left hepatectomy, the stomach adheres to the liver cut surface, which can cause unwanted gastric stasis [12-14]. The underlying mechanisms of gastric stasis after left hepatectomy are different from those of the usual laparotomy-associated adhesive ileus possibly due to rightward dislocation of the stomach after left hepatectomy and adhesion of the stomach to the cut surface of the rem- nant right liver [14].

The majority of patients who underwent extensive left-sided LN dissection were destined to receive adjuvant chemotherapy at 4 to 6 weeks after surgery. Thus, recovery of oral intake is essential to endure cytotoxic adjuvant chemotherapy. Postoperative delayed gastric emptying can deteriorate patients' quality of life because they have to restrict oral intake for a few weeks after surgery or keep an NGT until recovery of gastric motility [3]. Such gastric motility disorder also has a negative effect as it can significantly delay the start of adjuvant chemotherapy. Considering the poor prognosis of ICC patients with LN metastasis, gastric stasis and its associated degradation of quality of life can be considered as serious surgical complications.

To prevent left-sided LN dissection-associated gastric stasis, we 
have performed pyloroplasty as a preemptive procedure during resection of ICC with left-sided LN metastasis [3]. Prolonged gastric stasis universally happened in patients who had undergone extensive dissection of the gastric lesser curvature in our previous case series [3]. In contrast, gastric stasis occurred in 30\% after preemptive application of pyloroplasty in the present study. Considering that temporary gastric stasis can occur in $10 \%$ to $20 \%$ of patients who have undergone simple left hepatectomy without LN dissection [12-14], the actual incidence of gastric stasis after preemptive pyloroplasty was around $20 \%$ in the present study.

Two patients with gastric stasis were successfully treated with supportive care. However, one patient showed serious gastric paresis, which might be associated with vagotomy. The occurrence of chronic symptomatic gastric paresis has been reported to be $3 \%$ to $5 \%$ in patients following vagotomy and antrectomy [15]. Intravenous administration of erythromycin can improve the initial phase of solid meal gastric emptying in such patients [15]. However, erythromycin has not been available for a long time in our institution. Thus, we searched for alternative agents. Although metoclopramide and bethanechol were reported to increase gastric motor activity in the patients with diabetic and post-vagotomy gastroparesis [16], they were ineffective in our patient. Azithromycin is a macrolide like erythromycin. These two drugs can accelerate the gastric emptying of adult patients with gastroparesis. Azithromycin has advantages of longer duration of action and better side-effect profile while lacking P450 interaction, over erythromycin [17]. We followed the azithromycin administration protocol used in an old woman with diabetic gastroparesis [18], in which gastrointestinal symptoms were significantly improved after 3 days of treatment. Surprisingly, our patient showed noticeable improvement after only one session of azithromycin injection, as like triggering the start of gastric motility. A literature review study has concluded that azithromycin might be an alternative prokinetic agent in gastroparesis [19].

Performance of conventional Heinke-Mikulicz type pyloroplasty is a simple procedure that takes only 10 minutes because the pylorus-antrum area is usually normal in the majority of patients with ICC. Preemptive pyloroplasty has been proven to be effective to prevent gastric stasis. Thus, we think that there is no reason to hesitate whether to perform pyloroplasty or not if vagus nerve injury-associated gastric stasis is anticipated.

This study has some limitations. First, this study was a small case series from a single center, thus high-volume multicenter studies are necessary. Second, this study was a retrospective study, thus a prospective clinical trial is needed to validate the effect of preemptive pyloroplasty.

In conclusion, we believe that preemptive pyloroplasty is an ef- fective surgical option to prevent gastric stasis in patients undergoing extensive left-sided LN dissection. Azithromycin appears to be a potent prokinetic agent in gastroparesis.

\section{Notes}

\section{Conflicts of interest}

No potential conflict of interest relevant to this article was reported.

\section{Funding}

None.

\section{Author contributions}

Conceptualization, Data curation, Visualization: SH; Methodology: EKJ, DHJ, YK; Writing-original draft: SH; Writing-review \& editing: all authors.

\section{ORCID}

Shin Hwang, https://orcid.org/0000-0002-9045-2531

Dong-Hwan Jung, https://orcid.org/0000-0001-5984-023X

Eun-Kyoung Jwa, https://orcid.org/0000-0003-4820-0039

Yumi Kim, https://orcid.org/0000-0002-2811-2106

\section{References}

1. Tsuji T, Hiraoka T, Kanemitsu K, Takamori H, Tanabe D, Tashiro S. Lymphatic spreading pattern of intrahepatic cholangiocarcinoma. Surgery 2001;129:401-7.

2. Morine Y, Shimada M, Utsunomiya T, Imura S, Ikemoto T, Mori $\mathrm{H}$, et al. Clinical impact of lymph node dissection in surgery for peripheral-type intrahepatic cholangiocarcinoma. Surg Today 2012;42:147-51.

3. Cho JW, Lee HW, Hwang S. Benefit of pyloroplasty to prevent gastric stasis in intrahepatic cholangiocarcinoma patients undergoing extensive left-sided lymph node dissection. Korean J Hepatobiliary Pancreat Surg 2016;20:32-7.

4. Lee A. Gastroparesis: what is the current state-of-the-art for evaluation and medical management?: what are the results? J Gastrointest Surg 2013;17:1553-6.

5. Stevens JE, Jones KL, Rayner CK, Horowitz M. Pathophysiology and pharmacotherapy of gastroparesis: current and future perspectives. Expert Opin Pharmacother 2013;14:1171-86.

6. Hwang S, Lee YJ, Song GW, Park KM, Kim KH, Ahn CS, et al. Prognostic impact of tumor growth type on 7th AJCC Staging System for intrahepatic cholangiocarcinoma: a single-center experience of 659 cases. J Gastrointest Surg 2015;19:1291-304. 
7. Nathan H, Aloia TA, Vauthey JN, Abdalla EK, Zhu AX, Schulick RD, et al. A proposed staging system for intrahepatic cholangiocarcinoma. Ann Surg Oncol 2009; 16:14-22.

8. Clark CJ, Wood-Wentz CM, Reid-Lombardo KM, Kendrick ML, Huebner M, Que FG. Lymphadenectomy in the staging and treatment of intrahepatic cholangiocarcinoma: a population-based study using the National Cancer Institute SEER database. HPB (Oxford) 2011;13:612-20.

9. Adachi T, Eguchi S. Lymph node dissection for intrahepatic cholangiocarcinoma: a critical review of the literature to date. J Hepatobiliary Pancreat Sci 2014;21:162-8.

10. Ribero D, Pinna AD, Guglielmi A, Ponti A, Nuzzo G, Giulini SM, et al. Surgical approach for long-term survival of patients with intrahepatic cholangiocarcinoma: a multi-institutional analysis of 434 patients. Arch Surg 2012;147:1107-13.

11. de Jong MC, Nathan H, Sotiropoulos GC, Paul A, Alexandrescu $\mathrm{S}$, Marques $\mathrm{H}$, et al. Intrahepatic cholangiocarcinoma: an international multi-institutional analysis of prognostic factors and lymph node assessment. J Clin Oncol 2011;29:3140-5.

12. Lo CM. Complications and long-term outcome of living liver donors: a survey of 1,508 cases in five Asian centers. Transplantation 2003;75(3 Suppl):S12-5.

13. Hwang S, Lee SG, Lee YJ, Sung KB, Park KM, Kim KH, et al. Lessons learned from 1,000 living donor liver transplantations in a single center: how to make living donations safe. Liver Transpl 2006;12:920-7.

14. Kinoshita A, Takatsuki M, Hidaka M, Soyama A, Eguchi S, Kanematsu T. Prevention of gastric stasis by omentum patching after living donor left hepatectomy. Surg Today 2012;42:816-8.

15. Kendall BJ, Chakravarti A, Kendall E, Soykan I, McCallum RW. The effect of intravenous erythromycin on solid meal gastric emptying in patients with chronic symptomatic post-vagotomy-antrectomy gastroparesis. Aliment Pharmacol Ther 1997; 11:381-5.

16. Malagelada JR, Rees WD, Mazzotta LJ, Go VL. Gastric motor abnormalities in diabetic and postvagotomy gastroparesis: effect of metoclopramide and bethanechol. Gastroenterology 1980;78:286-93.

17. Larson JM, Tavakkoli A, Drane WE, Toskes PP, Moshiree B. Advantages of azithromycin over erythromycin in improving the gastric emptying half-time in adult patients with gastroparesis. J Neurogastroenterol Motil 2010;16:407-13.

18. Sutera L, Dominguez LJ, Belvedere M, Putignano E, Vernuccio L, Ferlisi A, et al. Azithromycin in an older woman with diabetic gastroparesis. Am J Ther 2008;15:85-8.

19. Potter TG, Snider KR. Azithromycin for the treatment of gastroparesis. Ann Pharmacother 2013;47:411-5. 